15.

\title{
SIBLING COMPETITION AND THE EVOLUTION OF BROOD SIZE AND DEVELOPMENT RATE IN BIRDS
}

\author{
Robert E. Ricklefs \\ Department of Biology, University of Missouri-St. Louis, St. Louis, MO 63121-4499, USA \\ (ricklefs@umsl.edu)
}

\begin{abstract}
Parents and their offspring may come into conflict over both the level of food provisioning to the brood and the distribution of food among nestlings within a brood. Sibling competition within a brood favours nestlings whose rapid development increases their competitive status. This may in turn reduce the overall fitness of parents by increasing the food requirements of individual nestlings. Parents can reduce conflict with their offspring by reducing the number of young in each brood and by hatching their eggs asynchronously, so that competitive status is determined by age and not by growth rate. Under these circumstances, benefits of slow development, possibly including a more responsive immune system or delayed senescence, may also result in slower growth and reduced food requirements of nestlings. Future studies of parentoffspring relationships should include evolutionary responses to competition between siblings and parental mechanisms for controlling these responses.
\end{abstract}

\section{INTRODUCTION}

Nestlings of altricial species of birds beg to obtain food from their parents, which respond to solicitation from their nestlings (Henderson 1975; Bengtsson \& Rydén 1983; Whittingham \& Robertson 1993; Ottosson et al. 1997). Experimentally increasing the intensity of nestling solicitation, for example by adding young to nests, depriving nestlings of food or playing begging 
vocalizations, generally stimulates parents to increase provisioning of food to the nest (Hussell 1988). This solicitation-response system should ensure that nestlings are optimally nourished with respect to maximizing their inclusive fitness and that parents distribute food among their offspring optimally with respect to their personal fitness. Parents and their offspring, however, may come into conflict over the allocation of food because of the basic asymmetry of genetic relationship between a nestling and its sibling (Trivers 1974). Godfray $(1991,1995)$ demonstrated that parents and their offspring could achieve a joint evolutionarily stable strategy with respect to solicitation and delivery of food. He did not, however, investigate situations in which nestlings compete directly within a brood, which has the potential to reduce evolutionary fitness of both parent and offspring by selecting traits, such as growth rate, that are useful in direct competition, but which reduce fitness overall.

In this chapter, I consider the effects of sibling competition on the growth rate and food requirements of nestlings and the consequences of variation in growth rate on the fitness of parents and their offspring. I also examine the more general relationship between food requirements of nestlings and parental provisioning and fitness, and the ways in which adults can adapt their parental strategies in response. Moreover, if parents were programmed behaviourally to satisfy nestling demands, this responsiveness could under stressful circumstances reduce lifetime reproductive success, particularly when nestlings acquire additional food at a low marginal return in personal fitness. Even when nestlings do not solicit or accept resources beyond the intake that is optimal for their parents (Cotton et al. 1996), reduced parental fitness could result from a mismatch between the demands of the brood and the capacity of the parents to supply the needs of their nestlings. Although such circumstances could arise in several ways, the most important may be unpredictable variation in the food supply. If females adjusted their brood size to average feeding conditions, periods of below average food availability could lead to undernourishment of the nestlings and excessive stress or risk to the parents as they try to satisfy nestling demands.

\section{FOOD REQUIREMENT AND PARENTAL PROVISIONING}

The food requirements of a brood are the product of the requirements of individual nestlings and the number of nestlings per brood. Individual requirements depend on many factors, including growth rate, heat production 
to regulate body temperature and activity at the nest (Dunn 1980; Weathers et al. 1997). Furthermore, requirements are not absolute. Rather, above a minimum threshold required for survival, additional food consumption by nestlings is thought to contribute incrementally to fitness at a diminishing rate.

Presumably, the female parent controls the number of nestlings per brood, while growth rate and activity are primarily strategies of nestlings. Due to the basic asymmetry of genetic relationships among siblings (Trivers 1974), nestlings and their parents may come into conflict with respect to such nestling adaptations as growth rate and such parental adaptations as brood size and responsiveness to solicitation. Rapid growth benefits nestlings directly by reducing time-dependent mortality, which may be high during the period of nestling development (Ricklefs 1969, 1984; Trivers 1974; Bosque \& Bosque 1995; Martin 1995). Moreover, rapid growth may improve the competitive position of a nestling within the brood when it achieves large size at an early age (Magrath 1990; Stouffer \& Power 1990). Rapid growth and consequent high food requirements may indirectly influence the inclusive fitness of a particular nestling by reducing the number of its siblings, either within its own brood or over the lifetime of its parents (Konarzewski et al. 1998). Selection on nestling traits always tends to maximize nestling inclusive fitness, regardless of costs to parental lifetime reproductive success.

Figure 1 shows how a mismatch between the requirements of the brood and the ability of parents to provide food could reduce parental fitness. This mismatch would most often arise owing to uncertainty regarding the state of the environment and of hatching success. Food shortage relative to demand influences fitness along two paths. The one to the right in Figure 1 is based on the direct link between nestling solicitation and parental response, which, in times of food shortage, can stress the parent and reduce lifetime reproductive success (Ottosson et al. 1997). This pathway presumes that parents do not withhold care from their offspring even when failing to do so reduces their own survival and future reproduction. That is, a parent's drive to satisfy its offspring's needs overrides its physiological assessment of its own personal condition. The evidence for this is strong, but not unanimous. Food deprivation usually leads to increased food solicitation by nestlings (Hussell 1988; Price \& Ydenberg 1995; Christe et al. 1996; Leonard \& Horn 1998), although the competitive environment may also influence the intensity of solicitation by a particular nestling (Leonard et al. 2000; Roulin et al. 2000; A. Roulin this volume) and nestlings low in the size hierarchy of a brood may passively endure starvation (M. Konarzewski personal communication). Brood enlargement experiments often show enhanced offspring production (Linden \& Møller 1989; Dijkstra et al. 1990) and reduced survival or future 
fecundity in parents (Dijkstra et al. 1990; Cucco \& Malacarne 1995; Jacobsen et al. 1995). Sometimes, however, adults appear to rear larger broods without adverse effects (Ydenberg \& Bertram 1989; Congdon 1990).

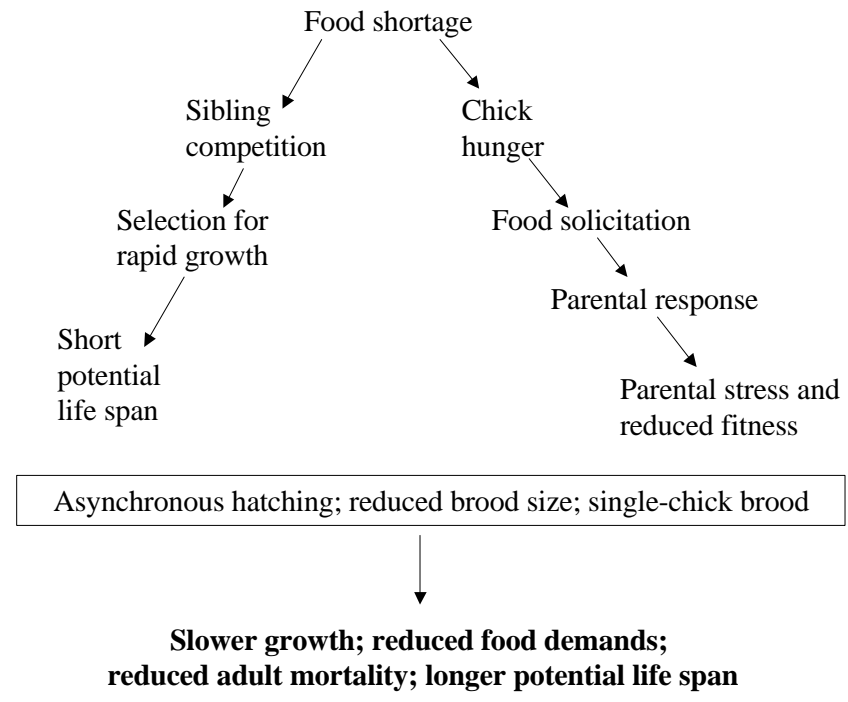

Figure 1. Food shortages influence fitness by imposing stress on adults mediated by nestling solicitation and parental response (right-hand path), and by the evolution of rapid growth through sibling competition (left-hand path). Reduced brood size and asynchronous hatching can eliminate parent-offspring conflict over the distribution of food and increase both parental fitness and the inclusive fitness of the nestlings.

The path to the left in Figure 1 is based on the advantage of size in sibling competition, which selects strongly for rapid growth. This can be shown by the observation that species with single-nestling broods have slower growth rates than those with multi-nestling broods (Werschkul \& Jackson 1979; Ricklefs 1982). Rapid growth rate increases the rate of optimal resource provisioning per nestling (Ricklefs 1984; Klaassen \& Bech 1992) and presumably forces parents to reduce brood size when food is limited. Rapid growth is also correlated in comparative analyses with reduced maximum potential life span (Ricklefs \& Scheuerlein 2001), perhaps through connections between early development and ageing (Ricklefs 1993). Models 
of the relationship between fitness and growth rate suggest that there is little conflict between parent and offspring when the primary cause of selection for rapid growth is time-dependent mortality (Konarzewski et al. 1998). Direct competition between broodmates, in contrast, can apply strong selection to increase the rate of growth, even in the absence of strong time-dependent mortality. In this case, small changes in growth rate can have large effects on fitness that are independent of surviving time-dependent mortality factors. Because fitness depends on growth rate relative to broodmates, selection will tend to increase growth rate continuously until it is limited by physiological or other intrinsic factors or until it is constrained by inclusive fitness considerations (Briskie et al. 1994; Anderson \& Ricklefs 1995). This high growth rate increases food demands on the parents without providing compensating increases in brood survival.

\section{PARENTAL CONTROL OVER PARENT-OFFSPRING CONFLICT}

Is the parent bird helpless in its conflict with its own nestlings? Several responses would seem to be possible. Parents can reduce the effect of nestling hunger on parental fitness by (1) moderating their responsiveness to nestling solicitation; (2) reducing brood size to reduce sibling competition and parentoffspring conflict, as well as parental stress; and (3) manipulating the competitive hierarchy among nestlings so that no advantage accrues from intense begging or rapid growth.

Can parents distribute food among nestlings in a manner that maximizes the total recruitment from the brood as a whole rather than the probability of recruitment of any particular individual nestling? To do so, the parent would have to discriminate between the stronger begging signals of better fed larger nestlings and the weaker signals of smaller but hungrier nestlings, in order to prevent first-hatched nestlings from controlling the distribution of food within the brood. Quantitative models have shown that parents can increase their personal fitness by providing better nourished nestlings with less food than needed to maximize a nestling's inclusive fitness, and using the balance to increase the survival of more poorly nourished siblings (Forbes 1993; Anderson \& Ricklefs 1995). Several studies indicate that parents of some species preferentially feed smaller, less dominant nestlings (Stamps et al. 1985; Leonard \& Horn 1996; Krebs et al. 1999).

By reducing the size of their brood, parents can bring the food requirements of the nestlings into line with the parents' abilities to provide food. Adjusting 
brood size can maximize the personal fitness of the parent in the context of parent-offspring conflict, but it does not directly diminish conflict produced by nestling adaptations. That is, growth rate and activity are still selected to maximize the inclusive fitness of the individual nestling at the expense of parental fitness. When brood size is reduced so much that nestlings no longer compete for resources, and thus do not conflict with parents over allocation of food, parental fitness may be reduced below what it would be when food is allocated optimally among nestlings in a larger brood.

When parents create conditions that minimize the selective advantage of sibling competition, nestlings may then reduce growth rate and other competitive traits to obtain other personal or inclusive fitness benefits. Slower growth also may increase the personal fitness of the parent in parallel with that of their nestlings, through larger potential brood size or reduced stress. In general, when there is no sibling competition, there is no parent-offspring conflict over the distribution of resources within a brood. Parents of many species appear to reduce the fitness consequences of sibling competition within broods by reducing brood size, so that there are adequate resources for all nestlings, and by asynchronous hatching of the nestlings. The latter mechanism determines the nestling dominance hierarchy by age (i.e. size) beyond the ability of nestling adaptations to change an individual's status within the brood (Ricklefs 1993). Both mechanisms are under parental control and provide the adult with potentially effective means of reducing the adverse consequences of parent-offspring conflict when parents are generally responsive to nestling solicitation. These mechanisms are consistent with variation in growth rates among species (Werschkul \& Jackson 1979; Ricklefs 1982, 1993) and with the observation that many species can rear more offspring than they normally do (Ydenberg \& Bertram 1989).

\section{Responsiveness to Nestling Solicitation}

Becoming less responsive to nestling solicitation has its own dangers in terms of inadequately providing for nestlings through mismatched solicitationresponse behaviour. I presume here that nestling solicitation honestly signals the degree to which its optimal nutritional level is satisfied at any particular time (Godfray 1991; Cotton et al. 1996). The optimal level should be adjusted to maximize the inclusive fitness of the nestling, although this may not correspond to the maximum personal fitness of the parent. I also presume that parents are selected to use the information concerning the nutritional state of their offspring to allocate food in a manner that maximizes their own personal 
fitness. In doing so, a parent may favour an undernourished nestling more than is desirable for the dominant, better nourished nestling(s) in a brood. This preference establishes selection on marginally nourished nestlings to overemphasize their poor condition, in a classic case of parent-offspring conflict (Johnstone 1996).

I can see no way for families to resolve this conflict other than through nestling begging hyperbole. A well-nourished nestling has no selection upon it to acquire additional resources and therefore begging signals should honestly indicate lack of hunger (Godfray 1995). Yet, when two hungry nestlings coexist in a brood and signal with the same relationship between begging intensity and undernourishment, discerning parents will allocate food between the nestlings to maximize parental fitness at the expense of maximizing the inclusive fitness of any one individual nestling. Thus, when nestlings compete strongly and relatively evenly within a brood, selection should drive the intensity of begging to progressively higher levels, restrained only when costs of extremely intense begging increase rapidly (Johnstone 1996). When nestling solicitation is inflated by conflict among offspring, it should be difficult for parents to discern the true nutritional level of their nestlings and to allocate food so as to maximize their own personal fitness. Note that inflation is not the same as dishonesty when there is no within-brood competition (Godfray 1995). What signal would be used, if relative body size (or age) cannot accurately predict the position of a nestling in the competitive hierarchy of a brood and thus its likely level of undernourishment (Harper 1986)?

\section{A Model of Sibling Competition}

A simple model relating nestling personal fitness to food provisioning illustrates the fitness consequences for parents and offspring of different levels of food allocation within a brood (Parker et al. 1989; Forbes 1993; Anderson \& Ricklefs 1995). Let $X$ be the food acquired by a nestling, and define the nestling's fitness by $W=1-X^{c}$, where $c[>0]$ determines how rapidly fitness increases with an increase in food acquisition above a threshold level of $X=1(W=0)$. This function increases at a progressively slowing rate from $W=0$ at a threshold level of food provisioning $(X=1)$ to an asymptote of $W=1$ at high values of $X$. Given this relationship between fitness and food consumption, when $R$ is the total food delivered to a two-nestling brood and $q$ is the proportion of $R$ consumed by the dominant A nestling, the inclusive fitness of the A nestling $\left(I_{A}\right)$ is maximized when: 


$$
\hat{q}=\frac{1}{1+2^{-\frac{1}{c+1}}}
$$

This result pertains so long as the fitness of the A nestling when it dominates its sibling $(q=1)$ is less than its inclusive fitness when it shares food, that is, $W_{A(q=1)}<I_{A}$, and the food share of the B nestling is greater than 1, that is, (1 q) $R>1$. Otherwise, the optimum proportion of food consumed by the A nestling is $\hat{q}=1$ (Anderson \& Ricklefs 1995). The value of $\hat{q}$ varies between 0.50 at $c$ approaching $\infty$ (fitness rises rapidly with increasing food consumption) and 0.67 as $c$ approaches 0 . When $c=3$, the A nestling should monopolize food $(q=1)$ at levels of delivery $(R)$ less than 2.76 and otherwise share food at level $\hat{q}=0.554$. These relationships are shown in Figure 2. This figure also shows the relative fitnesses of parents and offspring when the fitness of the parent is maximized ( $q=0.5$ when $R>2.47)$ and when the inclusive fitness of the A nestling is maximized ( $q=\hat{q}$ when $R>2.76$ ).

One can see that the consequences of parent-offspring conflict for the parents are substantial only for $R>2.47$ and approaching 2.76, at which point the parent loses about $20 \%$ of its potential fitness. Above $R=2.76$, the A nestling shares food and the maximum fitness difference is about $3 \%$. The differential between the inclusive fitnesses of the A and B nestlings at this point is about $11 \%$, which constitutes strong selection on the $\mathrm{B}$ nestling to improve its competitive position, especially compared to the relatively weak selection on parents to overcome parent-offspring conflict.

\section{Brood Size}

A second possibility for parents is to reduce the size of their broods, which increases the probability that nestlings are adequately nourished and reduces the probability that parents stress themselves in providing for their offspring. When conditions are relatively constant, parents could rear the young in a brood to a high level of fitness without reducing brood size substantially below the maximum they could nourish. Slightly undershooting the optimum reproductive output may be a good strategy when the costs of overshooting are high. When nestlings are provisioned at a high level, rapid growth is no longer favoured by sibling competition and personal and inclusive fitness factors favouring slow growth can predominate. Slower growth further 
reduces food requirements and may allow parents to increase the size of their broods. As discussed below, slow growth in multi-nestling broods is relatively common, especially among tropical birds.

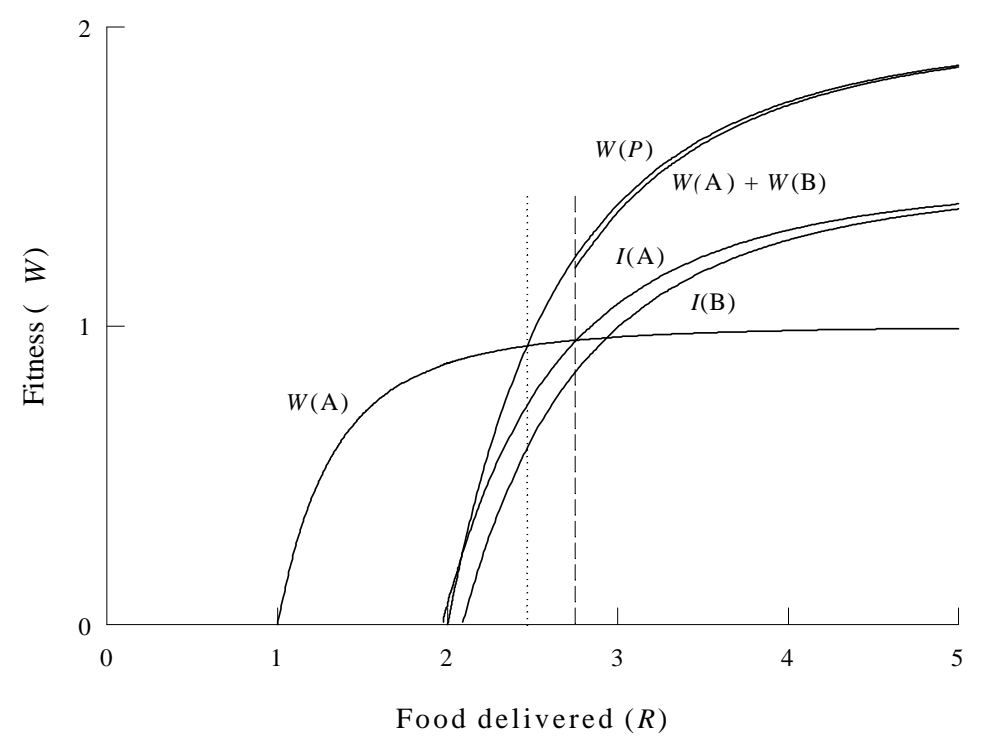

Figure 2. The relationship of various measures of fitness to food delivery to the nest, where $R=1$ is the minimum for reproductive success $>0$ (Anderson \& Ricklefs 1995). In this model, two nestlings (A and B) compete for food. $W(\mathrm{~A})$ is the fitness of the A nestling when it monopolizes all the food. I(A) is the inclusive fitness of the A nestling when it controls food allocation and maximizes its inclusive fitness. $I(\mathrm{~B})$ is the inclusive fitness of the $\mathrm{B}$ nestling when the A nestling maximizes its inclusive fitness. $W(\mathrm{P})$ is the combined fitness of the parents when food is distributed evenly between nestlings. $W(\mathrm{~A})+W(\mathrm{~B})$ is the combined fitness of the A and B nestlings when A shares food with B but maximizes its own inclusive fitness. The vertical dotted line is the lower limit of parent-offspring conflict over the allocation of food. To the left of this line only a single nestling is reared. The vertical dashed line indicates the point where the A nestling switches between food monopoly and food sharing with its younger B nestling, hence the upper bound of the region of maximum parent-offspring conflict.

A difficulty inherent in adjusting brood size is that the number of nestlings changes by integers, thus providing only a coarse adjustment (Ricklefs 1968). This is especially difficult for the transition between two nestlings and one nestling, where the productivity of the brood is nominally halved. However, this transition has occurred most commonly in pelagic seabirds and it has 
particularly interesting implications for parent-offspring relationships. Nestlings in broods of two compete for limiting resources and presumably they grow rapidly to maintain a size advantage in this competition. This results in a high food requirement during the growth period. When the food available to parents decreases below the level required by two rapidly growing nestlings, brood size must be reduced to one and reproductive output drops by one half. Following the two-nestling/one-nestling transition, however, sibling competition no longer exists within the brood and selection favours reduced growth rate, particularly where time-dependent mortality is low. This in turn allows the parents to exploit less rich or less rewarding food supplies, such as the open ocean at great distance from the nesting colony. The result is the situation typical of pelagic seabirds, with a single, slowly growing nestling and, in some cases, excess parental feeding capacity.

Once the single-nestling brood has been established, it may be difficult to return to a multi-nestling brood. As the food supply increases, either because of improving conditions or a change in the foraging ecology of the parents, individuals may pass the point at which they can rear two slowly growing nestlings. If a two-nestling brood is then produced, however, sibling competition between the nestlings can select for rapid nestling growth rate and food requirements, in which case the parents may no longer be able to nourish their young adequately. Thus, phylogenetic comparisons should reveal more frequent cases of transition from two nestlings to one.

The distribution of clutch size on the phylogeny of Sibley and Ahlquist (1990) suggests that single-egg clutches were derived independently in many groups, including the tropicbirds (Phaethontidae), boobies (Sulidae), frigatebirds (Fregatidae), petrels (Procellariiformes), New World vultures and the hawks and eagles. However, more detailed phylogenies based on DNA sequencing indicate that single-nestling broods may also be ancestral in some lineages, as in the case of some cotingas (Prum et al. 2000) and the bluefooted (Sula nebouxii) and Peruvian boobies (Sula variegata) (Friesen \& Anderson 1997). Such a reversal might occur when a newly exploited food supply is much more abundant than the former food supply.

\section{Optimum Brood Size under Variable Food Conditions}

When conditions vary unpredictably from one reproductive attempt to another, the point at which a parent sets its maximum reproductive potential (i.e. brood size) depends on the penalty imposed by trying to raise a large brood under poor feeding conditions. When future reproductive success falls 
off rapidly as adults are pushed to satisfy demands in excess of their capacity, parents should reduce brood size to correspond more closely to the poorest conditions they are likely to experience (Boyce \& Perrins 1987; Lalonde 1991). One consequence of such an adaptive response would be that adults could raise enlarged broods during most years and rarely show signs of reproductive stress.

We can see this effect in a simple model in which the capacity of the parents to deliver food to their brood $(R)$ varies from season to season and adult survival, nestling survival, or both depend on the ratio of nestling food demand $(D)$, which is proportional to brood size, to $R$. In this model, $R$ is lognormally distributed with the mean of $\log R$ equal to 0 (that is, $R=1$ ). Survival decreases with the demand/resource ratio according to $S=\exp (-$ $\left.a[D / R]^{b}\right)$. We define fitness as adult survival, plus recruitment. Parent survival is the product of $S_{N R}$, the component of survival unrelated to reproduction, and $S_{P}=f(D / R)$ as above. Recruitment is the product of food demand $D$ and offspring survival $S_{O}=f(D / R)$. When $a=0.5, b=3$, and both parents and nestlings suffer equally under a high demand/resource ratio, the optimum brood size is less than the mean resource availability and it decreases as the reproduction-independent survival increases (Figure 3). Using a standard deviation of $\log R$ of 0.3 (a factor of \pm 2.0 ), the optimum brood size is about $1 \mathrm{SD}$ unit below the mean when $S_{N R}=0.50$. Thus, resources would exceed demand in $87 \%$ of seasons and would be twice the demand in $9 \%$ of seasons. This effect increases as the non-reproductive survival increases. This model could be made more complete by including a threshold of resource abundance below which adults abandon their broods (Erikstad et al. 1998). The simpler model, however, makes the point that brood size may often be smaller than parents can rear in a particular season.

\section{Brood Reduction}

An alternative to setting a low brood size is facultative brood reduction (Lack 1954; Ricklefs 1965; O'Connor 1978; Mock \& Forbes 1994). In this case, brood size is set close to the maximum number of offspring that parents can rear during good years. In bad years, young are selectively starved, beginning with the youngest in the brood, until brood demand is brought into line with the ability of the parents to provide food. The problem with this strategy is that when parents cannot control their response to food solicitation, they are subjected to the constant physiological stress and risk of predation caused by having to provide for an overly large brood, even though the size of the brood 
may decrease over time. If this is the case, then examples of brood reduction become difficult to explain except during unusually poor feeding conditions.
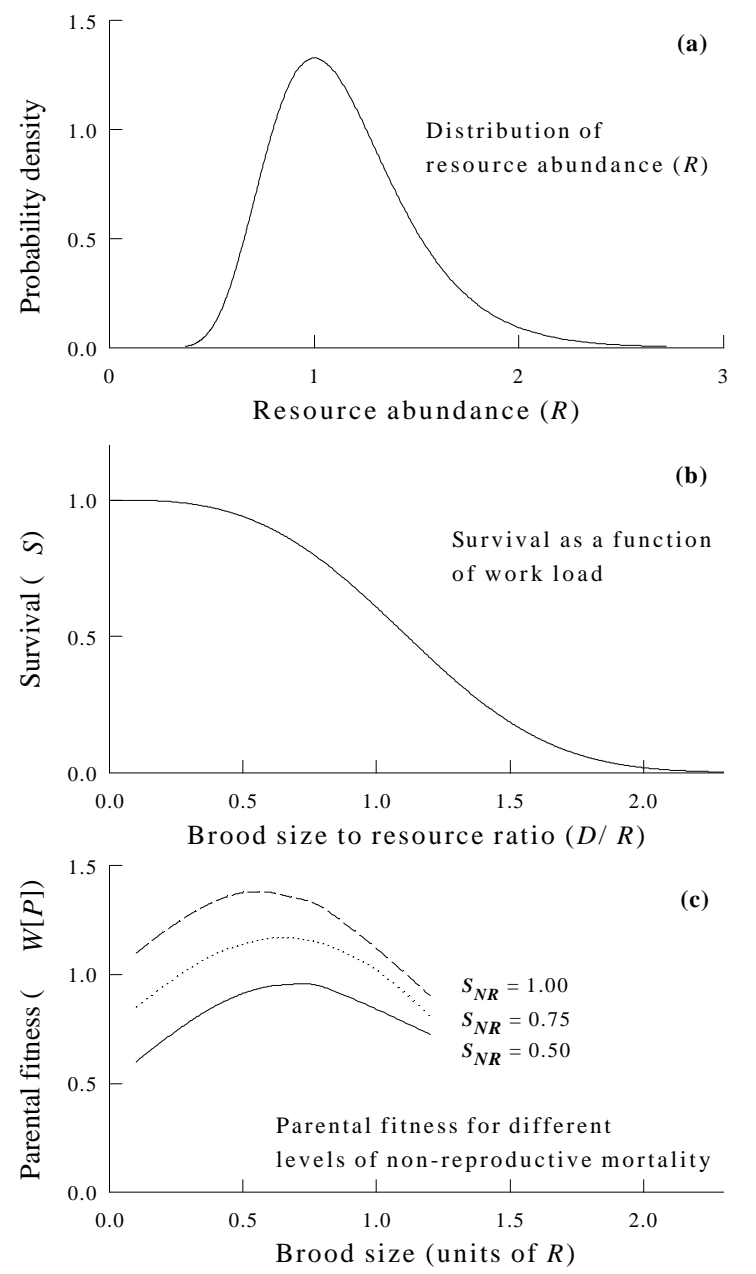

Figure 3. (a) Probability density distribution of resource abundance when resources are distributed log-normally with mean $0(R=1)$ and $\mathrm{SD}=0.3$. (b) Survival of adult or nestling according to the expression $S=\exp \left(-a[D / R]^{b}\right)$ when $a=0.5$ and $b=3$. (c) Parental fitness is the sum of adult survival plus number of offspring reared with the survival curves in (b) applied to both nestlings and adults and brood size scaled to units of $R$ under fluctuating environmental conditions (a). Curves in (c) are shown for different levels of survival due to factors unrelated to reproduction $\left(S_{N R}\right)$. 


\section{Manipulation of the Competitive Hierarchy}

The third general mechanism available to parents is to manipulate the competitive hierarchy among nestlings so the nestling cannot gain a fitness advantage through increased competitive advantage. Parents can accomplish this by establishing a rigid competitive hierarchy through asynchronous hatching (Anderson 1989; Lessells \& Avery 1989). Competitive success within a brood is determined primarily by body size (Hahn 1981; Bryant \& Tatner 1990; Stouffer \& Power 1990; Mock \& Parker 1997). By hatching the brood asynchronously, parents can establish size differences between nestlings that cannot be overcome by rapid embryonic or postnatal growth (Ricklefs 1993). This mechanism applies most clearly to complete asynchrony within the brood, but may also be effective when only a part of the brood hatches asynchronously (Stouffer \& Power 1990). By beginning full incubation after the first egg is laid, parents cause the nestlings to hatch in the order of laying at intervals equal to the laying interval. The spread between hatching can thus be increased by increasing the time between successive eggs. Although a single day is the most common laying interval in birds, intervals of two or more days are common, particularly among species in which the nestlings hatch asynchronously.

Ricklefs (1993) noted a strong association between the length of the incubation period and hatching asynchrony. He suggested that if size differences between nestlings could not be made up by variation in growth rate among nestlings, there would be no selective advantage to rapid growth resulting from increased competitive ability within a brood. Consequently, whatever advantages resulted from slow embryonic development would predominate and one would observe the evolution of long incubation periods. Because prolonged incubation provides no immediate benefit to either the nestling or the parent, and confers many disadvantages, Ricklefs (1993) suggested that a substantial benefit must be obtained through longer potential life span or higher reproductive rate. Potential mechanisms by which this could be accomplished would be to use the longer developmental period to build a better immune system (Ricklefs 1992) or nervous system. Direct evidence bearing on these hypotheses is not available.

The same argument pertains to nestling growth rate as to embryonic growth rate. Asynchronous hatching can eliminate the fitness consequences of variation in postnatal growth rate in a competitive environment and allow advantages of slow growth to predominate. In addition to the possibility of building a higher quality individual, slow growth also reduces food requirements. This may benefit the parent by allowing an increase in brood 
size, reduced stress of reproduction, or the exploitation of marginal food resources. It may also benefit the nestling directly by reducing the probability of undernourishment during poor feeding conditions, and indirectly by increasing inclusive fitness, that is, the number of siblings reared. When nestlings cannot increase their personal fitness through greater competitive ability, inclusive fitness of the nestling approaches the personal fitness of the parent and parent-offspring conflict diminishes or perhaps even disappears.

The foregoing arguments suggest that slow postnatal development and reduced parent-offspring conflict should occur when single nestlings are reared and when nestlings hatch asynchronously. There are few exceptions to the strong association between single-nestling broods and slow embryonic and postnatal growth (Werschkul \& Jackson 1979; Ricklefs 1982, 1993). Asynchronous hatching and slow embryonic and postnatal growth are also strongly associated (Ricklefs 1993; R.E. Ricklefs unpublished data). Indeed, exceptions to the general pattern in fact support the hypothesis or highlight alternative mechanisms for controlling sibling competition and parentoffspring conflict. Several groups of birds have asynchronous hatching and rapid postnatal growth. Without exception, these are either brood parasites (Cuculiformes, Buphagus, Molothrus, Viduinae), the hosts of brood parasites, or communal nesters (Coliidae). Where unrelated nestlings occur together in the same nest there is no kin-selected restraint upon competition through inclusive fitness effects. In this case, the most effective strategy for the parent and its nestling is to hatch as soon as possible by beginning incubation with the first egg laid and to grow as rapidly as possible.

Exceptions to the general pattern also occur in the opposite direction, that is, slow development with synchronous hatching. Ricklefs (1993) found that several groups of birds had synchronous hatching and long incubation periods: Alcedinidae (kingfishers), Criniferinae (go-away birds), Acanthisittidae (riflemen), Pipridae (manakins) and Acanthizini (thornbirds). None of these groups is well known, but detailed studies of the common kingfisher (Alcedo atthis) and rifleman (Acanthisitta chloris) found little or no starvation within broods (Gray 1969; Boag 1982). Thus, it is possible that food supplies for these species are predictable and adults have reduced brood size enough to eliminate sibling competition for food.

The general picture that emerges from comparative studies is that parents manipulate sibling competition to reduce development rate and increase personal fitness under two circumstances. The first, employed by pelagic seabirds, large raptorial birds, and a few tropical species (e.g. cotingas and some doves), enables parents to exploit marginal food resources. Reducing brood size to one nestling eliminates sibling competition and allows for the 
evolution of slow development. This in turn reduces demand for food delivery and allows parents to utilize even more marginal food resources. Somewhat surprising is the absence of transitional stages having two-nestling broods, asynchronous hatching, and slow development. Reducing clutch size produces a decreased food requirement quickly and reliably. Asynchronous hatching followed by selection for slow development may require a long period of evolution, at the beginning of which there is no reduction in food requirement, unless strong sibling hierarchies reduce the intensity of food solicitation and direct competition between nestlings.

The second circumstance occurs among generally long-lived species of primarily tropical birds, including most of the Coraciiformes, all Psittaciformes and Apodiformes, and some suboscine South American Passeriformes. Ricklefs (1993) argued that the primary advantage of long incubation periods in these groups is based on a link between slow embryonic development and long potential life span. Slow postnatal development would convey a further advantage to parents by reducing the food requirements of their nestlings. One of the chief difficulties of this hypothesis is that the benefits resulting from asynchronous hatching do not appear immediately, but follow many generations of evolution of slow growth rate. Incubation period is phylogenetically conservative, most of the variance in incubation period residing at the ordinal level (Ricklefs 1993). This difficulty would largely disappear if asynchronous hatching and slow development were ancestral, but there is no clear indication that this is so. The basal, non-raptorial altricial land birds, the Piciformes, uniformly have synchronous hatching, rapid embryonic development, and moderate to rapid post-hatching growth.

\section{THE TRANSITION BETWEEN LIFE HISTORIES WITH AND WITHOUT PARENT-OFFSPRING CONFLICT}

Parent birds can reduce or eliminate sibling competition and parent-offspring conflict by reducing the size of the brood, hatching the eggs asynchronously or both. Because changes in development rate evolve slowly, the primary advantages to smaller brood size and asynchronous hatching must be realized through reduced reproductive stress on the adults resulting from reduced demand at the nest. By itself, complete asynchronous hatching may reduce food requirement by reducing competitive behaviour within the brood as a direct consequence of a nestling's position within a size hierarchy. A size hierarchy within the brood may also help adults to distribute food optimally among nestlings under the range of food delivery rates in which parent- 
offspring conflict would be strong (see Figure 2). Subsequent advantages of slow development to the parent or nestling could be realized over longer evolutionary periods.

These considerations raise the question of why many species lack mechanisms to reduce sibling competition and parent-offspring conflict. Where potential adult life span is short owing to high mortality unrelated to reproduction, benefits of higher reproductive success from large broods may outweigh costs of additional adult mortality, and brood size may increase. Even with larger brood sizes, however, complete asynchronous hatching would appear to convey advantages when it facilitates brood reduction and reduces the costs of competition between nestlings. Balancing these benefits are several potential costs, including management of a brood having a spread of ages, especially at the time of fledging. In addition, sibling competition selects for rapid development, which may increase the fitness of parents under high time-dependent nestling mortality or where breeding seasons are short (Ricklefs 1984). Thus, under some circumstances, parents may use sibling competition to promote adaptations that increase their personal fitness at the expense of inclusive fitness of individual nestlings.

\section{FUTURE DIRECTIONS}

The study of parent-offspring interactions would benefit from more detailed modelling of the fitness consequences of asynchronous hatching and reduced brood size when evolutionary responses of nestling growth rate are included as factors that influence nestling food requirements and feed back on parental fitness through food provisioning rates. Such studies should be combined with additional empirical comparisons and experimental analyses of the relationship between parent and offspring fitness under varying food requirements and satisfaction of those requirements. Analysis of parentoffspring relationships in a phylogenetic context might provide insights into evolutionary transitions in brood size and hatching asynchrony. Nestling begging and parental responses should also parallel the control of sibling competition by parents, which could be addressed by comparative studies of begging in species with synchronous and asynchronous hatching. Where sibling competition and active begging attract predators, parents might be expected to control sibling competition even at the expense of brood size.

A major related problem is to understand the significance of slow development rates in some tropical species in spite of high time-dependent mortality of eggs and nestlings. Little is known of the dependence of food 
requirements on growth rate or the potential fitness benefits of slower growth. Although the evolution of slow growth with strongly asynchronous hatching is relatively straightforward, its occurrence in a few cases with synchronous hatching is puzzling. One might predict that such species would exhibit a high mean and a low within-brood variance in offspring fitness, indicating that young cannot improve their personal fitness through enhanced within-brood competition. Finally, empirical studies of brood-size-dependent nesting success and adult survival under varying conditions might shed light on reduced brood size as an strategy to improve lifetime reproductive success.

\section{ACKNOWLEDGEMENTS}

This chapter benefited from the insightful comments and suggestions of Marty Leonard, Kate Lessells and Jon Wright. The author's research is supported in part by NIH grant AG16895-01.

\section{REFERENCES}

Anderson, D.J. 1989. The role of hatching asynchrony in siblicidal brood reduction of two booby species. Behavioral Ecology and Sociobiology 25, 363-368.

Anderson, D.J. \& Ricklefs, R.E. 1995. Evidence of kin-selected tolerance by nestlings in a siblicidal bird. Behavioral Ecology and Sociobiology 37, 163-168.

Bengtsson, H. \& Rydén, O. 1983. Parental feeding rate in relation to begging behavior in asynchronously hatched broods of the great tit Parus major. An experimental study. Behavioral Ecology and Sociobiology 12, 243-251.

Boag, D. 1982. The Kingfisher. Dorset: Blandford Press.

Bosque, C. \& Bosque, M.T. 1995. Nest predation as a selective factor in the evolution of developmental rates in altricial birds. American Naturalist 145, 234-260.

Boyce, M. \& Perrins, C.M. 1987. Optimizing great tit clutch size in a fluctuating environment. Ecology 68, 142-153.

Briskie, J.V., Naugler, C.T. \& Leech, S.M. 1994. Begging intensity of nestling birds varies with sibling relatedness. Proceedings of the Royal Society of London, Series B 258, 73-78.

Bryant, D.M. \& Tatner, P. 1990. Hatching asynchrony, sibling competition and siblicide in nestling birds: studies of swiftlets and bee-eaters. Animal Behaviour 39, 657-671.

Christe, P., Richner, H. \& Oppliger, A. 1996. Begging, food provisioning, and nestling competition in great tit broods infested with ectoparasites. Behavioral Ecology 7, 127-131.

Congdon, B.C. 1990. Brood enlargement and post-natal development in the black noddy Anous minutus. Ети 90, 241-247.

Cotton, P.A., Kacelnik, A. \& Wright, J. 1996. Chick begging as a signal: are nestlings honest? Behavioral Ecology 7, 178-182.

Cucco, M. \& Malacarne, G. 1995. Increase of parental effort in experimentally enlarged broods of pallid swifts. Canadian Journal of Zoology 73, 1387-1395.

Dijkstra, C., Bult, A., Bijlsma, S., Daan, S., Meijer, T. \& Zijlstra, M. 1990. Brood size manipulations in the kestrel (Falco tinnunculus): effects on offspring and parent survival. Journal of Animal Ecology 59, 269-285. 
Dunn, E.H. 1980. On the variability of energy allocation of nestling birds. The Auk 97, 19-27.

Erikstad, K.E., Fauchald, P., Tveraa, T. \& Steen, H. 1998. On the cost of reproduction in long-lived birds: the influence of environmental variability. Ecology 79, 1781-1788.

Forbes, L.S. 1993. Avian brood reduction and parent-offspring "conflict". American Naturalist 142, 82-117.

Friesen, V.L. \& Anderson, D.J. 1997. Phylogeny and evolution of the Sulidae (Aves: Pelecaniformes): a test of alternative modes of speciation. Molecular Phylogenetics and Evolution 7, 252-260.

Godfray, H.C.J. 1991. Signalling of need by offspring to their parents. Nature 352, 328-330.

Godfray, H.C.J. 1995. Signaling of need between parents and young: parent-offspring conflict and sibling rivalry. American Naturalist 146, 1-24.

Gray, R.S. 1969. Breeding biology of rifleman at Dunedin. Notornis 16, 5-22.

Hahn, D.C. 1981. Asynchronous hatching in the laughing gull: cutting losses and reducing rivalry. Animal Behavior 29, 421-427.

Harper, A.B. 1986. The evolution of begging: sibling competition and parent-offspring conflict. American Naturalist 128, 99-114.

Henderson, B.A. 1975. Role of the chick's begging behavior in the regulation of parental feeding behavior of Larus glaucescens. Condor 77, 488-492.

Hussell, D.J.T. 1988. Supply and demand in tree swallow broods: a model of parentoffspring food-provisioning interactions in birds. American Naturalist 131, 175-202.

Jacobsen, K.O., Erikstad, K.E. \& Sæther, B.E. 1995. An experimental study of the costs of reproduction in the kittiwake Rissa tridactyla. Ecology 76, 1636-1642.

Johnstone, R.A. 1996. Begging signals and parent-offspring conflict: do parents always win? Proceedings of the Royal Society of London, Series B 263, 1677-1681.

Klaassen, M. \& Bech, C. 1992. Resting and peak metabolic rates of arctic tern nestlings and their relations to growth rate. Physiological Zoology 65, 803-814.

Konarzewski, M., Kooijman, S.A.L.M. \& Ricklefs, R.E. 1998. Models for avian growth and development. In: Avian Growth and Development. Evolution within the Altricial-Precocial Spectrum (Ed. by J.M. Starck \& R.E. Ricklefs). Oxford: Oxford University Press.

Krebs, E.A., Cunningham, R.B. \& Donnelly, C.F. 1999. Complex patterns of food allocation in asynchronously hatching broods of crimson rosellas. Animal Behaviour 57, 753-763.

Lack, D. 1954. The Natural Regulation of Animal Numbers. Oxford: Clarendon Press.

Lalonde, R.G. 1991. Optimal offspring provisioning when resources are not predictable. American Naturalist 138, 680-686.

Leonard, M. \& Horn, A. 1996. Provisioning rules in tree swallows. Behavioral Ecology and Sociobiology 38, 341-347.

Leonard, M.L. \& Horn, A.G. 1998. Need and nestmates affect begging in tree swallows. Behavioral Ecology and Sociobiology 42, 431-436.

Leonard, M.L., Horn, A.G., Gozna, A. \& Ramen, S. 2000. Brood size and begging intensity in nestling tree swallows. Behavioral Ecology 11, 196-201.

Lessells, C.M. \& Avery, M.I. 1989. Hatching asynchrony in European bee-eaters Merops apiaster. Journal of Animal Ecology 58, 815-835.

Linden, M. \& Møller, A.P. 1989. Cost of reproduction and covariation of life history traits in birds. Trends in Ecology and Evolution 4, 367-371.

Magrath, R.D. 1990. Hatching asynchrony in altricial birds. Biological Reviews 65, 587-622.

Martin, T.E. 1995. Avian life history evolution in relation to nest sites, nest predation and food. Ecological Monographs 65, 101-127. 
Mock, D.W. \& Forbes, L.S. 1994. Life-history consequences of avian brood reduction. The Auk 111, 115-123.

Mock, D.W. \& Parker, G.A. 1997. The Evolution of Sibling Rivalry. Oxford: Oxford University Press.

O'Connor, R.J. 1978. Brood reduction in birds: selection for fratricide, infanticide and suicide? Animal Behavior 26, 79-96.

Ottosson, U., Backman, J. \& Smith, H.G. 1997. Begging affects parental effort in the pied flycatcher, Ficedula hypoleuca. Behavioral Ecology and Sociobiology 41, 381-384.

Parker, G.A., Mock, D.W. \& Lamey, T.C. 1989. How selfish should stronger sibs be? American Naturalist 133, 846-868.

Price, K. \& Ydenberg, R. 1995. Begging and provisioning in broods of asynchronouslyhatched yellow-headed blackbird nestlings. Behavioral Ecology and Sociobiology 37, 201-208.

Prum, R.O., Rice, N.H., Mobley, J.A. \& Dimmick, W.W. 2000. A preliminary phylogenetic hypothesis for cotingas (Cotingidae) based on mitochondrial DNA. The Auk 117, 236-241.

Ricklefs, R.E. 1965. Brood reduction in the curve-billed thrasher. Condor 67, 505-510.

Ricklefs, R.E. 1968. On the limitation of brood size in passerine birds by the ability of adults to nourish their young. Proceedings of the National Academy of Sciences USA 61, 847-851.

Ricklefs, R.E. 1969. An analysis of nesting mortality in birds. Smithsonian Contributions to Zoology 9, 1-48.

Ricklefs, R.E. 1982. Some considerations on sibling competition and avian growth rates. The Auk 99, 141-147.

Ricklefs, R.E. 1984. The optimization of growth rate in altricial birds. Ecology 65, 1602-1616.

Ricklefs, R.E. 1992. Embryonic development period and the prevalence of avian blood parasites. Proceedings of the National Academy of Sciences USA 89, 4722-4725.

Ricklefs, R.E. 1993. Sibling competition, hatching asynchrony, incubation period, and lifespan in altricial birds. Current Ornithology 11, 199-276.

Ricklefs, R.E. \& Scheuerlein, A. 2001. Comparison of aging-related mortality among birds and mammals. Experimental Gerontology 36, 845-857.

Roulin, A., Kölliker, M. \& Richner, H. 2000. Barn owl (Tyto alba) siblings vocally negotiate resources. Proceedings of the Royal Society of London, Series B 267, 459-463.

Sibley, C.G. \& Ahlquist, J.E. 1990. Phylogeny and Classification of Birds. New Haven: Yale University Press.

Stamps, J., Clark, A.B., Arrowood, P. \& Kus, B. 1985. Parent-offspring conflict in budgerigars. Behaviour 94, 1-40.

Stouffer, P.C. \& Power, H.W. 1990. Density effects of asynchronous hatching and brood reduction in European starlings. The Auk 107, 359-366.

Trivers, R.L. 1974. Parent-offspring conflict. American Zoologist 14, 249-264.

Weathers, W.W., Hodum, P.J. \& Anderson, D.J. 1997. Is the energy cost of begging by nestling passerines surprisingly low? The Auk 114, 133.

Werschkul, D.F. \& Jackson, J.A. 1979. Sibling competition and avian growth rates. Ibis 121, 97-102.

Whittingham, L.A. \& Robertson, R.J. 1993. Nestling hunger and parental care in red-winged blackbirds. The Auk 110, 240-246.

Ydenberg, R.C. \& Bertram, D.F. 1989. Lack's clutch size hypothesis and brood enlargement studies on colonial seabirds. Colonial Waterbirds 12, 134-137. 

